\title{
Changes That Have Occurred in the Surgical Treatment Preference for Non-emergent Kidney Stones During the COVID-19 Pandemic: Six Months of Clinical Experience in A Tertiary Referral Centre
}

\author{
(1) Ali Furkan Batur, (1) Mustafa Gülmen, (1) Kadir Böcü, (1) Murat Gül, (1) Mehmet Kaynar, (1) Özcan Kılıç, (1) Serdar Göktaş \\ Selçuk University Faculty of Medicine, Department of Urology, Konya, Turkiye
}

What's known on the subject? and What does the study add?

The coronavirus disease 2019 pandemic has affected many of our treatment approaches due to the excessive overload it brings to the health system. Our study found that we operated on much less elective kidney stones during this pandemic process. Besides, the preoperative hydronephrosis rates were higher in patients who underwent retrograde intrarenal surgery. We anticipate that the future reflection of these findings will be a confrontation with more complicated patients and the interruption of residents' surgical training.

\begin{abstract}
Objective: Owing to the outbreak of the coronavirus disease-2019 (COVID-19), treatment approaches are reshapened because of the excessive load on the health system. This study aimed to investigate the differences in our surgical approach to kidney stone cases between the COVID-19 period and pre-COVID-19 period.

Materials and Methods: Patients who underwent retrograde intrarenal surgery (RIRS) or percutaneous nephrolithotripsy (PNL) for kidney stones in our clinic between March 11, 2020, and September 11, 2020, and those in the same period 1 year ago were included in the study. Demographic information, laboratory data, stone characteristics, treatment choices and results were evaluated comparatively.

Results: A total of 39 patients in the COVID-19 period (group 1) and 93 patients in the pre- COVID-19 period (group 2) underwent kidney stone surgery. While RIRS was performed to 32 of the patients in group 1, PNL was applied to seven of the patients in group 2 . RIRS was performed to 70 patients in group 2, and PNL was applied to 23 of them. While the RIRS/PNL ratio was 4.5 in group 1, it was 3.04 in group 2. During the COVID-19 period, the number of patients who underwent PNL has decreased significantly.

Conclusion: The number of elective surgery cases has significantly decreased during the COVID-19 pandemic. It is predicted that the incidence of organ loss will increase due to complications and delayed treatment. Moreover, interruption of the residents' surgical training secondary to the decreasing number of cases is one of the pandemic's critical drawbacks.
\end{abstract}

Keywords: COVID-19, RIRS, PNL, kidney stone

\section{Introduction}

The prevalence of urinary stones varies between $1 \%$ and $20 \%$, and in recent years, this value has increased in many parts of the world. A study reported that it has increased by more than $37 \%$ in the last 20 years (1). The European Association of Urology (EAU) guidelines recommended percutaneous nephrolithotripsy (PNL) and retrograde intrarenal surgery (RIRS) as the first choice in the surgical treatment of kidney stones $>20 \mathrm{~mm}$ and $<10 \mathrm{~mm}$, respectively. Both methods are recommended for stone sizes between $10 \mathrm{~mm}$ and $20 \mathrm{~mm}$ (1). Although the success rate and stone-free rate (SFR) in a single session are lower than that with $\mathrm{PNL}$, the use of RIRS has increased in recent years for stones $>20 \mathrm{~mm}$, owing to the growing experience, which results in low complication rates and shorter hospital stay $(2,3)$.

December 2019 was considered the starting time point of the coronavirus disease 2019 (COVID-19) pandemic. In Turkey, the first COVID-19 cases have been identified on March 10, 2020. A

Correspondence: Ali Furkan Batur MD, Selçuk University Faculty of Medicine, Department of Urology, Konya, Turkiye

Phone: +90 3322244995 E-mail: alifurkanbatur@gmail.com ORCID-ID: orcid.org/0000-0001-7945-7326

Received: 17.12 .2020

Accepted: 25.12 .2020

Cite this article as: Batur AF, Gülmen M, Böcü K, Gül M, Kaynar M, Kılıc Ö, Göktaş S. Changes That Have Occurred in the Surgical Treatment Preference for Non-emergent Kidney Stones During the COVID-19 Pandemic: Six Months of Clinical Experience in A Tertiary Referral Centre. J Urol Surg 2021;8(2):86-91.

๑Copyright 2021 by the Association of Urological Surgery / Journal of Urological Surgery published by Galenos Publishing House. 
pandemic was also declared by the World Health Organisation on March 11, 2020 (4). The COVID-19 pandemic has led to radical decisions that affected all aspects of daily practices in many fields, especially in the health system (5).

With the allocation of most of the health resources to patients with COVID-19, changes were necessary in our urology practice, as in all clinical branches, in accordance with the COVID-19 recommendation guide (6). Patients who developed anuria, sepsis due to stone-induced obstruction and renal failure were included in the emergency group. Immediate intervention with nephrostomy or Double-J (DJ) stent placement was recommended to this group. The high priority group included patients who would be clinically impaired if intervention is delayed by $>6$ weeks. The medium priority group includes cases where a 3-4 week delay in the intervention would cause clinical harm to the patient. The low priority group includes cases in which the patient is unlikely to experience complications even if the intervention was delayed for 6 months. As a result, cases of renal calyx stones were included in the lowest priority group. With these guidelines, the number of surgeries has decreased.

Consequently, it appears possible that we will encounter patients whose treatments were delayed and, therefore, whose disease will become more severe. As an overlooked aspect, we may see the negative indications of this pandemic on the future, as experience with difficult cases, which are a part of the theoretical and practical educations of residents, were disrupted in third-level referral and educational institutions.

With the above background, this study aimed (1) to compare outcomes of RIRS and PNL performed electively on renal stones during the COVID-19 period and 1-year pre-COVID-19 period, (2) to evaluate whether our approaches to similar cases of renal stones have changed and (3) to raise the issue of disruption in the education of the residents. With this study, we hope to draw a future projection with changing approach trends.

\section{Materials and Methods}

This retrospective study was approved by the institutional review board (decision number 2020/554), and the study protocol followed ethical standards.

Patients who underwent RIRS or PNL for kidney stones during the 6 months between March 11, 2020, and September 11, 2020, when the COVID-19 pandemic has started, and patients who had undergone the same operations in the same period exactly 1 year ago (between March 11, 2019 and September 11, 2019) were included in the study. Patients who needed urgent decompression with nephrostomy or DJ stent due to obstruction and septic status, with acute kidney damage, were under 18 years of age and were pregnant were excluded. Parameters such as age, gender, preoperative blood creatinine level, blood urea nitrogen level, kidney surgery history, extracorporeal shock wave lithotripsy history, preoperative hydronephrosis (HN) status, stone location and localisation, stone size and volume, presence or absence of accompanying ureteral stones, surgery option and residual stone after treatment were collected from our database and evaluated comparatively between the groups. Before surgery, all patients were assessed with urine analysis, urine culture, serum biochemistry, coagulation test and non-contrast computed tomography of the urinary system as a requirement of routine practice. The stone burden was calculated by the formula according to the EAU guideline (stone volume= length $\times$ width $\times$ depth $\times \pi \times 0.167)(7)$.

Both procedures were performed under general anaesthesia. RIRS was performed in the lithotomy position. A semi-rigid ureteroscope (Olympus Medical Systems, Hamburg, Germany) was routinely performed before flexible ureteroscopy in all patients for dilatation of the ureter and placement of a 0.035inch sensor guide wire. According to the surgeon's technical preference, all stones were dusted with $272 \mu \mathrm{m}$ Holmium YAG laser fibre (Dornier Medilas H 20 Laser, Wessling, Germany) until they were deemed small enough to pass spontaneously. A 4.8-Fr DJ stent (Cook Medical, Bloomington, IN, USA) was routinely placed in all patients. PNL was performed in the prone position. Mini PNL (Karl-Storz Medical, Tuttlingen, Baden-Württemberg, Germany) was applied to all patients as a part of routine practice. After 24-Fr dilatation in each patient, an Amplatz sheath (Cook Medical, Bloomington, IN, USA) was placed, and lithotripsy was performed with a pneumatic lithotripter $\left(\mathrm{EMS}^{\circledR}{ }^{\circledR}\right.$, Swiss Lithoclast ${ }^{\circledR}$ Master, Nyon, Switzerland). Stones were extracted with forceps. Malecot catheter was placed in every patient as a routine practice.

\section{Outcome Measures}

Primary outcome measures were the differences in treatment selections and stone characteristics between the COVID-19 period and pre-COVID-19 period.

\section{Statistical Analysis}

All statistical analyses were performed using the IBM Statistical Package for the Social Sciences version 22 (IBM SPSS Statistics for Windows, Chicago, IL, USA). While quantitative values are given by mean \pm standard deviation for parametric data, quantitative values for nonparametric data are provided by the median [minimum-maximum (min-max)]. Data of categorical variables are presented as numbers and percentages. ShapiroWilk and $0-0$ plots were used to check the normality of the variables. Data were expressed as mean \pm standard deviation (range, min-max) or median (interquartile range) for continuous variables and described as counts (n) and percentages (\%) for categorical variables. Independent t-test, Mann-Whitney U 
test and chi-square test were used to evaluate differences in parameters between the groups. A p-value of less than 0.05 was considered significant.

\section{Results}

While 39 patients underwent surgery for kidney stones during the COVID-19 period (group 1), 93 patients underwent surgery during the pre-COVID-19 period (group 2). While 32 of the patients in group 1 had undergone RIRS, 7 of them had received PNL; 70 of the patients in group 2 had undergone RIRS, and 23 had received PNL. The difference was not significant $(p=0.396)$. While the RIRS/ PNL ratio was 3.04 in group 1, it was 4.5 in group 2.

Patients' demographic, stone and surgical characteristics are summarised in Table 1. While the median patient age in group 1 was 41 (14-75) years, this value was 49 (21-5) years in group 2, and the difference was not significant $(p=0.063)$. No difference was found in the gender distribution between group 1 and group $2(p=0.943)$.

A significant difference was found between the two groups in terms of the presence of $\mathrm{HN}(\mathrm{p}=0.01)$. While $9(23 \%)$ patients in group 1 did not have $\mathrm{HN}, 44$ (47\%) patients in group 2 did not have HN. A significant difference was found between the patients who underwent RIRS in group 1 and group 2 in terms of the presence of HN ( $p=0.015)$. Relatively more patients had HN in group 1 (78.1\% vs 52.9\%) than in group 2.

No difference was found in the preoperative blood urea nitrogen, blood creatinine, stone size and stone volume parameters between group 1 and group 2 (Table 1). Moreover, no difference in stone number was found between group 1 and group 2 ( $p=0.126)$. The number of patients with non-lower pole and lower pole localisation in both groups was comparable. No difference was found in terms of residual stones between the two groups $(p=0.185)$.

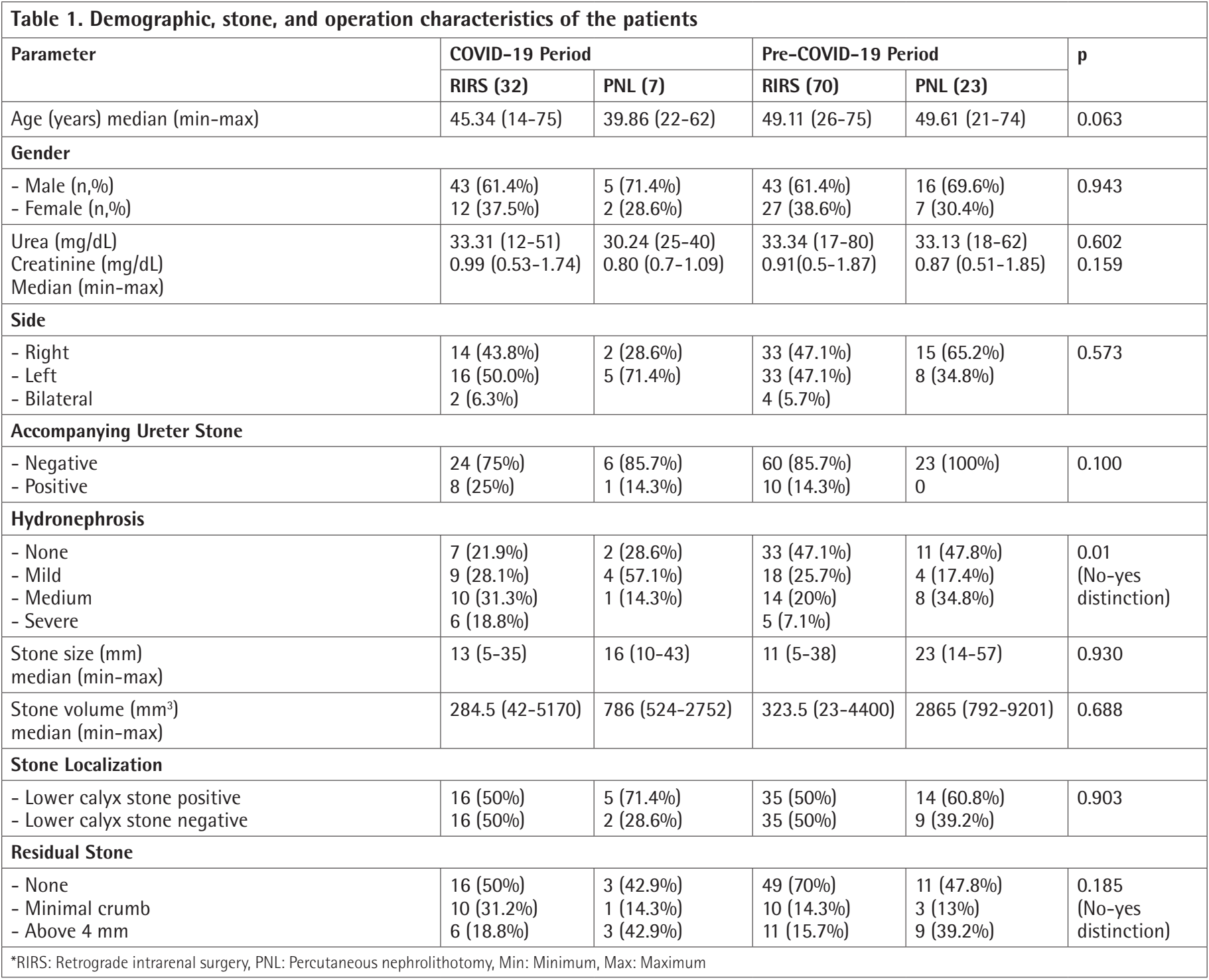


When the patients were evaluated separately according to the surgery groups, no significant difference was found between patients in group 1 and group 2 who underwent RIRS. Still, the stone size of patients who underwent RIRS during the COVID period tended to increase slightly (13 $\mathrm{mm}$ vs $11 \mathrm{~mm}$ ).

A significant difference was found between patients in group 1 and group 2 who underwent PNL and between patients with $786 \mathrm{~mm}^{3}$ versus $2865 \mathrm{~mm}^{3}$ of stone volume. During the COVID-19 period, the stone volume of patients with PNL decreased $(p=0.048)$ and the number of patients with PNL has reduced significantly.

\section{Discussion}

The unprecedented COVID-19 pandemic has affected health systems worldwide and revolutionised all medical practices. The number of cases that can be evaluated as elective has decreased significantly $(8,9)$. In this context, many national and international urology associations have published guidelines to clarify clinical and surgical priorities during the COVID-19 pandemic. However, it is still unclear how the priority will be determined in urinary stones. During this process, patients evaluated in the emergency group received interventions according to the EAU recommendation guide. An algorithm for approaching stone diseases was proposed by the EAU stone study group (10). According to this algorithm, nephrostomy or DJ stent intervention was recommended for patients with obstruction (HN) with decreased kidney function and patients with urinary tract infection and severe pain, even if the kidney function was normal. Surgical intervention is not recommended for the vast majority of patients without HN. Surgical drainage is recommended only in patients with urinary tract infection who have the risk of developing sepsis despite antibiotherapy and in patients whose pain persists despite analgesia (10).

No difference was found between the groups in our study regarding accompanying ureteral stones and stone localisation. However, $47 \%$ of our patients who underwent surgery during the COVID-19 period had HN. A significant difference was found between the groups in terms of the degree and presence of $\mathrm{HN}$. The increase in the severity of $\mathrm{HN}$ in patients who underwent surgery during COVID-19 may indicate that the patients' treatment was delayed. The increased incidence of $\mathrm{HN}$ is also considered an indicator that patients with $\mathrm{HN}$ are given priority, even if RIRS and PNL were considered.

Lei et al. (11) found that $41 \%$ of the patients with COVID-19 required intensive care and $20.5 \%$ of the patients who underwent elective surgery died even during the incubation period. For this reason, elective surgeries that may require follow-up in the intensive care unit during the postoperative period have been avoided, and less invasive options have been evaluated instead. Other reasons are as follows: use of hospitals as pandemic hospitals, decrease in the number of service beds, operating rooms and intensive care beds that can be used and patients do not present to the hospital due to the threat of COVID-19 transmission. In our study, when the COVID-19 period and pre-COVID-19 period were compared, the rate of RIRS was significantly increased, and the number of patients who underwent PNL decreased significantly.

Studies have indicated that the trend in kidney stones larger than $2 \mathrm{~cm}$ for which PNL is usually recommended has shifted to RIRS as it is less invasive, regardless of the COVID-19 period. The reason is that some PNL surgeries mostly require more than one session because the stones are scattered in different calices, resulting in severe renal damage. In these multiple entry cases, the risk of bleeding complications also increases (12-14). In these patients, RIRS is offered as a treatment option. However, owing to the efficiency of lithotripsy, SFR values are low in RIRS surgeries in stones $>2 \mathrm{~cm}$, and more than one surgical application may be required (1). A recent systematic review on renal stones $>2 \mathrm{~cm}$ showed a cumulative SFR of 91\% with 1.45 procedures/patient (15). When the stone volumes and localisation were evaluated in our study, no significant difference was found between the groups. However, the stone size of the patients who underwent RIRS during the COVID period increased slightly. In this case, many studies have demonstrated the possibility of a higher rate of residual stones due to RIRS on larger stones $(16,17)$. In our study, in the intragroup analysis of patients who underwent RIRS, no difference was found between the SFR rates.

With the new normalisation process after the COVID-19 pandemic, elective surgeries have been performed gradually since June 1, 2020. Surgical interventions that were delayed due to the clinics' inability to work at full capacity have accumulated over time. Patients with kidney stone represent a significant proportion of urological surgical cases that were postponed during the pandemic and thus still awaiting surgery. As future reflection, postponement of elective surgery will increase organ losses because of complications and delayed treatment (18). In this study, no difference was found between the blood urea nitrogen and creatinine levels in the renal function evaluation of the patients. However, more detailed examinations are required to evaluate the stone-related damage in the kidneys.

Furthermore, interruption of the residents' surgical training secondary to the decreasing number of cases in hospitals where third-level training is provided is one of the most significant drawbacks. Owing to the decreasing number of cases and quarantine process worldwide, especially in the USA, the surgical training gap has been eliminated by web-based platforms (19). In this process, web-based training programmes were more often organised in Turkey and in our clinic. With this change, there 
may be differences in the traditional education programmes of residents because surgery should be learned through practice.

\section{Study Limitations}

The retrospective design, postponement of elective cases during the pandemic and low number of patients included in the study because of the decreased patient admissions are the study's limitations. In addition, the 6-month period in which the patients included in the study who underwent surgery, included heterogeneous processes from full restriction to the new normalisation process. However, for prospective research, the pandemic must be over. The increase in COVID-19 cases during the transition to the normalisation process also interrupted elective surgical cases.

\section{Conclusion}

During the COVID-19 pandemic, many urological procedures were postponed. The less invasive option was preferred. Although elective surgeries have begun to be performed in a controlled manner with the new normalisation process, it is still uncertain how the accumulated cases will be resolved in the process. As future reflection, organ losses will increase because of complications and delayed treatment. Another critical issue is the delay in the practical training of surgical residents. As the pandemic period gets longer, the variety and number of elective cases performed by residents will decrease significantly.

\section{Ethics}

Ethics Committee Approval: This retrospective study was approved by the institutional review board (decision number: 2020/554), and the study protocol followed ethical standards.

Informed Consent: Retrospective study.

Peer-review: Externally peer-reviewed.

\section{Authorship Contributions}

Surgical and Medical Practices: A.F.B., K.B., Mur.G., M.K., Ö.K., S.G., Concept: A.F.B., Design: A.F.B., Data Collection or Processing: M.G., K.B., Analysis or Interpretation: A.F.B., M.G., Literature Search: A.F.B., Writing: A.F.B., M.G., M.K., Ö.K., S.G.

Conflict of Interest: No conflict of interest was declared by the authors.

Financial Disclosure: The authors declare that they have no relevant financial.

\section{References}

1. Türk C, Neisius A, Petrik A, Seitz C, Skolarikos A, Thomas K. EAU Guidelines on Urolithiasis. European Association of Urology 2018;69:475-482.
2. Karagöz MA, Erihan IB, Doluoğlu ÖG, Uğurlu Ç, Bağcıoğlu M, Uslu M, Sarıca $K$. Efficacy and safety of fURS in stones larger than $20 \mathrm{~mm}$ : is it still the threshold? Cent European J Urol 2020;73:49-54.

3. Palmero JL, Durán-Rivera AJ, Miralles J, Pastor JC, Benedicto A. Comparative study for the efficacy and safety of percutaneous nefhrolithotomy (PCNL) and retrograde intrarenal surgery (RIRS) for the treatment of 2-3,5 cm kidney stones. Arch Esp Urol 2016;69:67-72.

4. Jee Y. WHO International Health Regulations Emergency Committee for the COVID-19 outbreak. Epidemiol Health 2020;42:2020013.

5. McCloskey B, Zumla A, Ippolito G, Blumberg L, Arbon P, Cicero A, Endericks T, Lim PL, Borodina M; WHO Novel Coronavirus-19 Mass Gatherings Expert Group. Mass gathering events and reducing further global spread of COVID-19: a political and public health dilemma. Lancet 2020;395:10961099.

6. Ribal MJ, Cornford $P$, Briganti $A$, Knoll $T$, Gravas $S$, Babjuk $M$, Harding $C$, Breda A, Bex A; GORRG Group, Rassweiler JJ, Gözen AS, Pini G, Liatsikos E, Giannarini G, Mottrie A, Subramaniam R, Sofikitis N, Rocco BMC, Xie LP, Witjes JA, Mottet $N$, Ljungberg $B$, Rouprêt $M$, Laguna MP, Salonia $A$, Bonkat G, Blok BFM, Türk C, Radmayr C, Kitrey ND, Engeler DS, Lumen N, Hakenberg OW, Watkin N, Hamid R, Olsburgh J, Darraugh J, Shepherd R, Smith EJ, Chapple CR, Stenzl A, Van Poppel H, Wirth M, Sønksen J, N'Dow J; EAU Section Offices and the EAU Guidelines Panels. European Association of Urology Guidelines Office Rapid Reaction Group: An Organisationwide Collaborative Effort to Adapt the European Association of Urology Guidelines Recommendations to the Coronavirus Disease 2019 Era. Eur Urol 2020;78:21-28.

7. Türk C, Knoll T, Petrik A, Sarica K, Skolarikos A, Straub M, Seitz C. Guidelines on urolithiasis. Eur Assoc Urol 2015.

8. Heinze $A$, Umari $P$, Basulto-Martínez $M$, Suárez-Ibarrola $R$, Liatsikos $E$ Rassweiler J, Guven S, Gözen AS. Impact of COVID-19 on Clinical and Academic Urological Practice: A Survey from European Association of Urology Section of Uro-technology. Eur Urol Open Sci 2020;21:22-28.

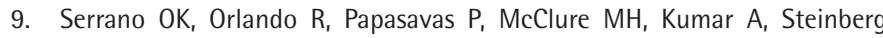
AC, Cohen JL, Shichman SJ, Singh RK, Sardella WV, Schipper BM. Getting back to work: A framework and pivot plan to resume elective surgery and procedures after COVID-19. Surg Open Sci 2021;4:12-18.

10. Tefik T, Guven S, Villa L, Gokce MI, Kallidonis P, Petkova K, Kiremit MC, Sonmez MG, de Lorenzis E, Eryildirim B, Sarica K. Urolithiasis Practice Patterns Following the COVID-19 Pandemic: Overview from the EULIS Collaborative Research Working Group. Eur Urol 2020;78:21-24.

11. Lei $S$, Jiang $F$, Su W, Chen $C$, Chen J, Mei W, Zhan LY, Jia Y, Zhang L, Liu D, Xia ZY, Xia Z. Clinical characteristics and outcomes of patients undergoing surgeries during the incubation period of COVID-19 infection. EClinicalMedicine 2020;21:100331.

12. De $\mathrm{S}$, Autorino R, Kim FJ, Zargar H, Laydner H, Balsamo R, Torricelli FC, Di Palma C, Molina WR, Monga M, De Sio M. Corrigendum re: "Percutaneous Nephrolithotomy Versus Retrograde Intrarenal Surgery: A Systematic Review and Meta-analysis" [Eur Urol 2015;67:125-37]. Eur Urol 2016;69:85.

13. El-Nahas AR, Shokeir AA, El-Assmy AM, Mohsen T, Shoma AM, Eraky I, ElKenawy MR, El-Kappany HA. Post-percutaneous nephrolithotomy extensive hemorrhage: a study of risk factors. J Urol 2007;177:576-579.

14. Michel MS, Trojan L, Rassweiler JJ. Complications in percutaneous nephrolithotomy. Eur Urol 2007;51:899-906.

15. Wu T, Duan X, Chen S, Yang X, Tang T, Cui S. Ureteroscopic Lithotripsy versus Laparoscopic Ureterolithotomy or Percutaneous Nephrolithotomy in the Management of Large Proximal Ureteral Stones: A Systematic Review and Meta-Analysis. Urol Int 2017:99:308-319.

16. Wang $Y$, Zhong B, Yang X, Wang G, Hou P, Meng J. Comparison of the efficacy and safety of URSL, RPLU, and MPCNL for treatment of large upper impacted ureteral stones: a randomized controlled trial. BMC Urol 2017;17:50. 
17. Geraghty R, Abourmarzouk O, Rai B, Biyani CS, Rukin NJ, Somani BK. Evidence for Ureterorenoscopy and Laser Fragmentation (URSL) for Large Renal Stones in the Modern Era. Curr Urol Rep 2015;16:54.

18. Amparore $D$, Campi $R$, Checcucci $E$, Sessa $F$, Pecoraro $A$, Minervini $A$, Fiori C, Ficarra V, Novara G, Serni S, Porpiglia F. Forecasting the Future of
Urology Practice: A Comprehensive Review of the Recommendations by International and European Associations on Priority Procedures During the COVID-19 Pandemic. Eur Urol Focus 2020;6:1032-1048.

19. Smigelski M, Movassaghi M, Small A. Urology Virtual Education Programs During the COVID-19 Pandemic. Curr Urol Rep 2020;21:50. 